\title{
ECCENTRICITY OF NETWORKS WITH STRUCTURAL CONSTRAINTS ${ }^{1}$
}

\author{
MATJAŽ KRNC \\ FAMNIT, University of Primorska, Koper \\ Faculty of Information Studies \\ Novo Mesto, Slovenia \\ e-mail: matjaz.krnc@upr.si \\ JEAN-SÉBASTIEN SEREni \\ Centre national de la recherche scientifique (C.N.R.S.) \\ ICube, CSTB, Strasbourg, France \\ e-mail: sereni@kam.mff.cuni.cz

\section{Riste ŠKrekovski} \\ Faculty of Information Studies, Novo Mesto \\ Faculty of Mathematics and Physics, University of Ljubljana \\ FAMNIT, University of Primorska, Koper, Slovenia \\ e-mail: skrekovski@gmail.com
}

AND

Zelealem B. Yilma

Carnegie Mellon University Qatar

Doha, Qatar

e-mail: zyilma@qatar.cmu.edu

\footnotetext{
${ }^{1}$ This work was made possible by Partenariat Hubert Curien Proteus [37455VB]; ARRS [BIFR-PROTEUS/17-18-009, P1-0383], Agence Nationale de la Recherche [anr 10 jcjc 0204 01], the European Commission through the InnoRenew CoE project (Grant Agreement \#739574) under the Horizon2020 Widespread-Teaming program and the Republic of Slovenia (Investment funding of the Republic of Slovenia and the European Union of the European regional Development Fund), and the generous support of the Qatar Foundation through Carnegie Mellon University in Qatar's Seed Research program. The statements made herein are solely the responsibility of the authors.
} 


\begin{abstract}
The eccentricity of a node $v$ in a network is the maximum distance from $v$ to any other node. In social networks, the reciprocal of eccentricity is used as a measure of the importance of a node within a network. The associated centralization measure then calculates the degree to which a network is dominated by a particular node. In this work, we determine the maximum value of eccentricity centralization as well as the most centralized networks for various classes of networks including the families of bipartite networks (twomode data) with given partition sizes and tree networks with fixed number of nodes and fixed maximum degree. To this end, we introduce and study a new way of enumerating the nodes of a tree which might be of independent interest.
\end{abstract}

Keywords: eccentricity, network, bipartite graph, complex network, maximum degree.

2010 Mathematics Subject Classification: Primary: 91D30, Secondary: 05C35, 68R10, 05C05.

\title{
REFERENCES
}

[1] P. Bonacich, Simultaneous group and individual centralities, Soc. Netw. 13 (1991) $155-168$. doi:10.1016/0378-8733(91)90018-O

[2] S.P. Borgatti and M.G. Everett, Network analysis of 2-mode data, Soc. Netw. 19 (1997) 243-269.

doi:10.1016/S0378-8733(96)00301-2

[3] A. Davis, B.B. Gardner and M.R. Gardner, Deep South: A Social Anthropological Study of Caste and Class (University of Chicago Press, Chicago, 1941).

[4] R. Dunbar, The social brain hypothesis, Evolutionary Anthropology 6 (1998) 178-190. doi:10.1016/S0378-8733(96)00301-2

[5] M.G. Everett, P. Sinclair and P. Dankelmann, Some centrality results new and old, J. Math. Sociol. 28 (2004) 215-227. doi:10.1080/00222500490516671

[6] K. Faust, Centrality in affiliation networks, Soc. Netw. 19 (1997) 157-191. doi:10.1016/S0378-8733(96)00300-0

[7] L.C. Freeman, A set of measures of centrality based on betweenness, Sociometry 40 (1977) 35-41. doi: $10.2307 / 3033543$

[8] L.C. Freeman, Centrality in social networks conceptual clarification, Soc. Netw. 1 (979) 215-239. 
[9] P. Hage and F. Harary, Eccentricity and centrality in networks, Soc. Netw. 17 (1995) $57-63$. doi:10.1016/0378-8733(94)00248-9

[10] C. Heuberger and S. Wagner, Maximizing the number of independent subsets over trees with bounded degree, J. Graph Theory 58 (2008) 49-68. doi:10.1002/jgt.20294

[11] C. Heuberger and S. Wagner, Chemical trees minimizing energy and Hosoya index, J. Math. Chem. 46 (2009) 214-230. doi:10.1007/s10910-008-9456-6

[12] A. Ilić, Eccentric connectivity index, in: Novel Molecular Structure Descriptors Theory and Applications II, Mathematical Chemistry Monographs 9, I. Gutman and B. Furtula (Ed(s)), (University of Kragujevac and Faculty of Science Kragujevac, 2010) 139-168.

[13] C. Jordan, Sur les assemblages de lignes, J. Reine Angew. Math. 70 (1869) 185-190. doi:10.1515/crll.1869.70.185

[14] M. Krnc, J.-S. Sereni, R. Škrekovski and Z.B. Yilma, Closeness centralization measure for two-mode data of prescribed sizes, Netw. Sci. 4 (2016) 474-490. doi:0.1017/nws.2016.14

[15] J.A. McHugh, Algorithmic Graph Theory (Prentice-Hall, Inc., Upper Saddle River, NJ, 1990).

[16] M.E.J. Newman, Networks: An Introduction (Oxford University Press, Oxford, 2010). doi:10.1093/acprof:oso/9780199206650.001.0001

[17] P. Sinclair, Betweenness centralization for bipartite graphs, J. Math. Sociol. 29 (2004) 25-31. doi:10.1080/00222500590889730

[18] P. Sinclair, Erratum: Betweenness centralization for bipartite graphs, J. Math. Sociol. 29 (2005) 263-264. doi:10.1080/00222500590961630

[19] P. Sinclair, Network centralization with the Gil Schmidt power centrality index, Soc. Netw. 31 (2009) 214-219. doi:10.1016/j.socnet.2009.04.004

[20] S. Wasserman and K. Faust, Social Network Analysis: Methods and Applications (Cambridge University Press, Cambridge, 1994). doi:10.1017/CBO9780511815478 
[21] D.B. West, Introduction to Graph Theory (Prentice Hall, Inc., Upper Saddle River, NJ, 1996).

[22] G. Yu, L. Feng and A. Ilić, On the eccentric distance sum of trees and unicyclic graphs, J. Math. Anal. Appl. 375 (2011) 99-107.

Received 18 April 2017

Revised 18 June 2018

Accepted 23 August 2018 